\title{
Brain morphological alteration and cognitive dysfunction in multiple system atrophy
}

\author{
Lihong Zhang ${ }^{1}$, Li Zhang ${ }^{2}$, Fang Xue ${ }^{1}$, Kathy Yue ${ }^{3}$, Haixin Peng ${ }^{4}$, Ya'nan Wu ${ }^{1}$, Ou Sha ${ }^{5}$, Lan Yang ${ }^{1}$, \\ Yan Ding ${ }^{6}$ \\ ${ }^{1}$ Department of Neurology, The Second Hospital of Hebei Medical University, Shijiazhuang 050000, China; ${ }^{2}$ Department of Anatomical and \\ Cellular Pathology, Prince of Wales Hospital, The Chinese University of Hong Kong, Hong Kong, China; ${ }^{3}$ School of Public Health, University of \\ Minnesota, Minneapolis, MN 55455, USA; ${ }^{4}$ Department of Food Science and Nutrition, Sichuan Agricultural University, Chengdu 611130, China; \\ ${ }^{5}$ Department of Anatomy, Histology and Developmental Biology, School of Basic Medical Sciences, Shenzhen University Health Science Centre, \\ Shenzhen 518060, China; ${ }^{6}$ Department of Neurology, Xuanwu Hospital, Capital Medical University, Beijing 100053, China
}

Correspondence to: Yan Ding. Department of Neurology, Xuanwu Hospital, Capital Medical University, 45 Changchun Street, Beijing 100053, China. Email: dingyandoctor@126.com.

\begin{abstract}
Multiple system atrophy (MSA) is a progressive neurodegenerative disease in adults, manifesting various clinical symptoms including autonomic nerve dysfunction, Parkinson's syndrome, cerebellar ataxia, and pyramidal sign. The clinical diagnosis and classification of MSA are mainly dependent on motion and non-motion symptoms, such as autonomic nerve dysfunction. In addition, an increasing amount of clinical and pathological evidence has shown that about half of the MSA patients exhibit distinct types and levels of cognitive dysfunction. However, cognitive dysfunction has not been included in the current diagnosis criteria of MSA. In most cases, it was even used as an exclusion criterion of MSA. Based on the neuroimaging, neuropathology and neuropsychology, this review summarized the morphological changes of the brain in the patients with MSA, and discussed possible brain regions that could be associated with cognitive impairment. The article may provide a theoretical basis for incorporating cognitive dysfunction into the criteria of MSA diagnosis.
\end{abstract}

Keywords: Multiple system atrophy (MSA); cognitive dysfunction; morphological change

Submitted Jul 10, 2018. Accepted for publication Oct 31, 2018.

doi: 10.21037/qims.2018.11.02

View this article at: http://dx.doi.org/10.21037/qims.2018.11.02

\section{Introduction}

Multiple system atrophy (MSA) is a sporadic, adult-onset and progressive neurodegenerative disorder. It was firstly proposed and named by Graham with clinical characteristics mainly including autonomic nervous dysfunction, Parkinsonism, cerebellar ataxia, and pyramidal signs (1-3). The result of the epidemiological survey demonstrated that the mean incidence of MSA was 0.6 to 0.7 cases per 100,000 person-years, with 3.4 to 4.9 cases per 100,000 person-years for the point prevalence (4). On average, symptoms appear after the age of 60 without a significant gender variance. The mean survival time of MSA patient ranges from 6 to
10 years, and in rare cases, a patient may survive for more than 15 years (4). So far, the causes of MSA are still unclear. No special treatment is available except symptomatic treatments (5). The symptoms and signs of MSA are rather diverse. The clinical diagnosis of MSA mainly depends on the case history and physical examination of the nervous system with neuropathological changes as the base for a final diagnosis (4). Currently, according to the motor symptoms, MSA can be divided into two subgroups: MSA with predominant Parkinsonism (MSA-P) and with predominant cerebellar ataxia (MSA-C) (1). Besides the classic motor symptoms, there are still a series of nonmotor features, such as autonomic failure, sleep disorders, 
cognitive disorders and emotion disorder, which may occur in every stage during MSA development. In around 20\% to $75 \%$ of patients, motor disturbances appear months or even years after they suffered from MSA, which makes it rather difficult for early diagnosis and causes misdiagnosis and delayed identification $(4,6)$.

In the 2008 consensus conference for MSA, the cognitive impairment was not involved as one of the indexes for diagnosis and the dementia was even considered as one of the eliminating indexes (1). However, along with the development of neuropsychology, neuropathology and neuroimaging, reports regarding MSA cases with the cognitive impairment have rapidly increased. According to clinical data analysis, about $1 / 3$ to $1 / 2$ of MSA patients suffered from a variety of cognitive impairments to a distinct extent (7-9). The autopsy results showed that $5 \%$ to $26 \%$ of MSA patients had dementia $(7,10,11)$. The occurrence of cognitive impairment in MSA patients performed differently in the length of time. Some appeared after seven years (12), while others took place in the early stage $(13,14)$, even as an initial symptom (14).

The aim of this article is to summarize recent studies, focusing on the link between brain structure, pathology change and cognitive impairment, for better understanding of the cognitive disorders in MSA patients.

\section{The main manifestations and assessment methods of cognitive impairment}

The key indicators of cognitive impairment include the dysfunctions in attention, memory, language, visuospatial recognition, frontal-executive abilities, etc. $(9,15)$. In these symptoms, frontal-executive dysfunction and memory deficit are the major indicators of cognitive impairment. Over $41 \%$ MSA patients have frontal-executive dysfunction, which is the most common type of cognitive impairment and belongs to frontal lobe syndrome $(7-9,16,17)$.

The primary assessments utilized for cognitive dysfunction testing of MSA patients were up to date, and was neuropsychological based. Currently, the two most common clinical detection tools are Mini-Mental State Examination (MMSE) and Montreal Cognitive Assessment (MoCA). MMSE is widely used for general patient population screening. Due to the lacking in measurement of executive functions, MMSE can barely discern mild cognitive impairment. MoCA has a higher sensitivity for detecting cognitive impairment caused by a subcortical impairment, as well as visuospatial and executive dysfunctions (18-20).

\section{Neuromorphology and cognitive impairment}

Currently, studies related to the brain structures of MSA patients with cognitive impairment often utilize the neuroimaging and neuropathology methods. Magnetic resonance imaging (MRI) is the most commonly used imaging technique $(21,22)$. Based on MRI, T1-weighted image (T1WI) and voxel-based morphometry (VBM), a study showed that the brain atrophy of MSA patients was mainly localized in the caudate nucleus, cerebellum, insular cortex, fusiform gyrus, inferior orbito-frontal gyrus, and superior temporal gyrus (23). Another study using T2-weighted MRI scans to diagnose a group of mixedtype MSA patients ( $\mathrm{n}=37$ vs. 16 controls) showed that the brain atrophy of patients occurred in the cerebellar cortex, pons, and putamen (24). In addition, a recent study with similar VBM revealed that compared to a control group, the significant reduced cerebellar volume and gray matter atrophy in the bilateral basal ganglia, cerebellum, temporal, and frontal cortex, as well as paracentral lobule, and parahippocampal gyrus were observed in the MSA-P group (25). Moreover, a study measured the volumetric MRIs of MSA patients via a novel automated segmentation software with a result illustrating that the volumes of cerebellum, thalamus, putamen, pallidum, hippocampus, and brainstem of the MSA group were much smaller than those of the control group; however, the volumes of ventricles, including the lateral ventricles, $3^{\text {rd }}$ and $4^{\text {th }}$ ventricles were all dilated (26). It is also reported recently that the cortical thinning in the front-temporoparietal regions and the volume of subcortical structures were significantly reduced in the 18 patients with MSA-C when compared to 50 healthy controls (27). Of note, microscopic examination followed by an autopsy revealed that in the MSA-P patients, the atrophy in putamen was obvious, it would extend to caudate nucleus in some severe cases and the substantia nigra became much whiter (28). In the MSA-C patients, the atrophy was primarily in the cerebellum, middle cerebellar peduncle (MCP), and pontine basal. The cerebellum atrophy was localized in the cortex. The white matter was remarkably decolored, and the pons locus coeruleus became paler. Furthermore, there was no atrophy in the cerebral cortex and thalamus in the both subgroups of MSA patients (28).

Based on the various technologies and assessment methods discussed above, it can be seen that the main 

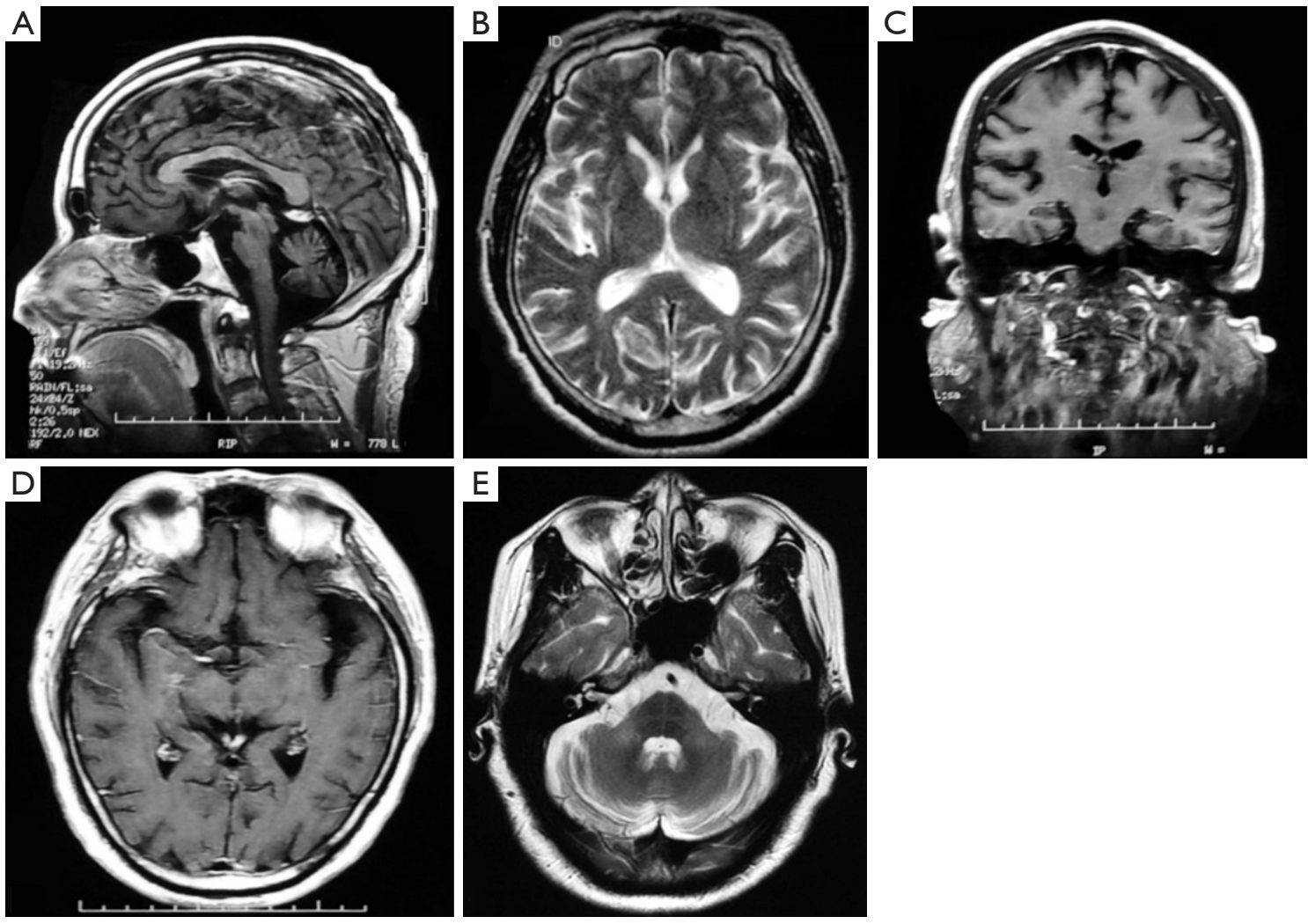

Figure 1 The brain atrophy profile of patients with MSA by MRI. (A) The brain T1-weight MRI detects brain stem and cerebellum were atrophic; (B) the brain T2-weight MRI shows atrophy of bilateral basal ganglia and putaminal slit sign; (C) the brain T1-weight MRI observes obvious atrophy of cerebral cortex and atrophy of hippocampus; (D) the brain T1-weight MRI reveals lateral fissure widening and temporal lobe atrophy; (E) the brain T2-weight MRI demonstrates the pons are atrophic, the cross sign can be seen in the pons, and the bilateral pons have symmetrical hyperintensity.

areas of brain atrophy of MSA patients take place in the basal ganglia, brainstem and cerebellum, as well as frontal and temporal lobes, though the cortex also shows imaging widespread atrophy. We summarize the MRI findings of patients with MSA admitted to Xuanwu Hospital of Capital Medical University and find that brain atrophy also accords with these characteristics (Figure 1A,B,C,D,E). The general observation of an autopsy strongly supports the results from neuroimaging.

\section{Neuropathology and cognitive impairment}

The classic pathological alterations in MSA patients include the formation of the central nervous system (CNS), $\alpha$-synuclein-positive glial cytoplasmic inclusions (GCIs), and neurodegenerative alterations in the striatonigral and olivopontocerebellar structures (1,29). Regarding the mechanism underneath the neuron degeneration and brain atrophy in MSA patients, it is commonly considered that the mutation of oligodendrocytes followed by the demyelination and the mutation of nerve cells is due to the formation of insoluble GCIs induced by an abnormally excessive $\alpha$-synuclein accumulation in the oligodendrocytes; or, the direct accumulation of $\alpha$-synuclein within nerve cells, forming neuronal cytoplasmic inclusions (NCIs), and the triggering of the death mechanism of the nerve cells $(3,30,31)$. Additional studies illustrated that in most areas of the brain, the density of GCIs correlates positively with neuronal cells loss. The number of GCIs increases gradually with the disease development $(31,32)$.

It has been reported that in MSA patients, GCIs are widespread in the white matter of CNS, the subcortical nucleus, and the cortex. With the most amount in the basal ganglion (caudate nucleus and putamen), pyramidal tract, corticocerebellar tracts, substantia nigra, pontine nuclei (including pontocerebellar tracts), medulla oblongata 
dorsal lid (including dorsal nucleus of vagus nerve), inferior olivary nucleus (including olivocerebellar fibers), and the white substance of cerebellum. There are also many GCIs in the motor cortex and globus pallidus, still some in the temporal lobe, superior frontal gyrus, hippocampus, amygdala, subthalamus, red nucleus, midbrain dense layer, and cerebellar dentate nuclei, most of which are neurodegenerative, and atrophic areas $(3,29,33)$. Another similar study found that there were more NCIs than GCIs in the substantia nigra, suggesting a tight correlation between the death of the substantia nigra neuron and NCIs (31).

Based on past studies, typical pathological changes in MSA-P and MSA-C patients are striatonigral degeneration (SND) and olivopontocerebellar atrophy (OPCA), respectively $(28,31)$. One study targeting $100 \mathrm{MSA}$ patients with semi-quantitative pathological analysis demonstrated that the neuron loss occurred mostly in the dorsolateral putamen, vermis cerebelli and substantia nigra, and less in the caudate nucleus. The neuron loss in the MSA patients with SND was mainly in the putamen, caudatum, pallidum and nigra. In the patients with OPCA, the loss mainly happened in pons, cerebellar hemisphere, vermis cerebelli and inferior olivary nucleus (31). Another report summarized the clinicopathological characteristics of 203 MSA patients from 108 publications and obtained similar results, in that the cerebellar impairment was induced by the degeneration of the Purkinje cells and dentate nucleus neurons (34). Interestingly, a study had also explored the brain metabolism of SND- and OPCA-types with fludeoxyglucose F18 positron emission tomography (PET), and identified that the lowest metabolism activity in these two types of MSA had occurred in the putamenpallidum complex, and cerebellum respectively. This is also corresponded to their pathological changes; moreover, the lower the metabolic activity, the more severe the clinical symptoms (35).

Even though the studies discussed above have demonstrated that the pathological changes were approximately consistent with the alterations of medical imaging usage in MSA patients, the pathological changes are more widespread. In some areas such as hippocampus, obvious atrophy can be detected via imaging methods; however, rare reports showed the decrease of neurons in these areas by pathology (3). Instead, in other areas, no significant atrophies can be detected by imaging, whereas many GCIs can be observed in the neurons by using pathological techniques, which may indicate that certain pathological alterations occur, before brain atrophy. Or, that
GCI induces the abnormalities in MSA patients via other molecular mechanisms (36).

\section{Possible mechanisms of cognitive impairment in MSA}

Currently, the mechanisms of cognitive impairment in MSA patients remain unknown, but the general consensus is that the atrophy of the cortex, the subcortical structure, and the cerebellum contribute to the generation of MSA $(3,9)$. According to the location of these pathological changes, there are three different hypotheses which attempt to explain the potential mechanism for developing MSA.

\section{The damage in the subcortical circuit causes signal conduction impairment}

Abundant subcortical afferent and efferent nerve fibres, together with basal ganglion-centric nucleus accumbens, inter-connect the cerebral cortex, brainstem and cerebellum as medium. This creates various closed circuit loops with distinct functions, including the cerebral cortexbasal ganglia-thalamus-cerebral cortex loop and the cerebral cortex-pons-cerebellum-thalamus-cerebral cortex loop. These loops establish the structural foundation of information processing in brain to execute motor and nonmotor related functions (37-39). According to the different functional areas that the projection fibers are connected to in the cerebral cortex, these circular structures are further divided into several independent circuit loops with their own functions. The one connecting to the frontal lobe is involved in cognitive functions such as working memory, learning ability, and execution. Basal ganglia in the loop mainly contains functional structures include: caudate nucleus, putamen, globuspallidus, reticular formation of brainstem, and subthalamic nucleus. Inside the cerebellum, the responsible structures are the dentate nucleus, and the intercalatus nucleus. Among which, efferent nerve fibers derived from the globuspallidus, reticular structure of substantia nigra, and dentate nucleus neurons primarily project into the frontal and temporal cortex. In addition, the properties and the impairment symptoms of the neurons from the globuspallidus, reticular structure of substantia nigra, and dentate nucleus are very similar to these ones from frontal lobe $(38,40,41)$.

The fiber tracking technique based on diffusion tensor imaging (DTI) is used widely to track human brain connectivity and cognitive impairment $(42,43)$. The results 
illustrated that there was a large mass of loop connections between corpus striatum and cerebral cortex, substantia nigra, brainstem, as well as cerebellum. The subcortical afferent fibers connecting with prefrontal lobe are derived from the head of caudate nucleus and putamen, which are consistent with evidence from neuroanatomy (44-48).

\section{The damage of the cerebral cortex-basal ganglia- thalamus-cerebral cortex circuit}

Based on the previous studies of neuroanatomy, neuropathology and neuroimaging, the classical opinions considering the cognitive impairment of MSA patients is mainly caused by the lesions of subcortical circuits related to cognitive function (49). The most significant pathological alteration of MSA-P patients is the degeneration of striatal cells (31). This further promotes damage of the cerebral cortex-basal ganglia-thalamus-cerebral cortex circuit, and cognitive impairment such as executive hypofunction, which is induced by the retardant of the afferent nerve of the corpus striatum to cerebral cortex especially frontal lobe $(9,15,25,39,50)$.

\section{The damage of the cerebral cortex-pons-cerebellum- thalamus-cerebral cortical circuit}

Many studies have shown that cerebellum lesions can result in the coexistence of non-motor symptoms, including both cognitive and emotional disorders. These are mainly characterized by the impairment of brain executive functions like language ability, spatial cognition, and personality alteration. This series of clinical behavioral changes has been named as, "cerebellar cognitive affective syndrome (CCAS)" (51).

A the previous study containing 20 patients with cerebellar injuries was primarily localized within the posterior lobe and vermis, which are responsible for cognitive and emotional disorders respectively, had illustrated that the deficits of patients were consistent with the ones due to impairment of cerebellar contact area. For example, prefrontal lobe injury induced executive dysfunction, parietal lobe injury induced visual spatial dysfunction, frontal and temporal lobes injury induced language disorder, cingulate gyrus and parahippocampal gyrus injuries induced an emotional disorder. These areas are linked to the cerebellum via the cerebrum-cerebellum loop and cerebellum-limbic system, such as hippocampus et al., and regulate cognitive function and emotional state together. In sum, it is possible that CCAS of patients was caused by the damage of a subcortical circuit loop that connects cerebellum and cerebral cortical projection area, leading to mis-transferring of essential signals $(37,38,40,41,51,52)$. A large spectrum of neuropsychological tests were performed to examine the IQ, attention, verbal and visuospatial memory, and the executive function of 20 patients with MSA-C. The results showed that those MSA-C patients had similar disordered performances in executive function, language and visuospatial memory as CCAS patients, suggesting there to be a similar mechanism (53).

\section{Primary cerebral cortex, especially the frontal lobes injuries}

Chang and his colleagues obtained brain atrophy patterns and cognitive changes of 10 MSA-C and 13 MSA-P patients in comparision with 37 healthy controls via MRI and neuropsychological tests. The following correlation analyses showed that memory deficits were correlated with prefrontal lobe significantly (23). In another study, Kawai et al determined cognitive dysfunction of the 21 MSA-C patients and 14 MSA-P patients by neuropsychological tests and regional brain blood flow measurement via SPECT analysis. It was proved that in the early stage, only MSA-P patients showed a obvious decrease of prefrontal lobe cerebral perfusion when compared with the healthy controls. This implied that cognitive disorder in MSA-P patients might be related to prefrontal lobe (15).

\section{Lesions from cerebral cortex and subcortical structure together contribute to the cognitive dysfunction in MSA patients}

Recently, high resolution MRI scanning technique was applied to examine brain atrophy patterns in 18 MSA-C patients, and 50 healthy control patients by Lee group (27). The correlation among different atrophy areas and between atrophy pattern and cognitive alteration were further checked by linear regression analysis. The results demonstrated that the atrophy in MSA-C patients were widespread in cerebral cortex, subcortical structure and cerebellum. These atrophy areas could develop independently without influence from each other, among which attention deficit was related to the atrophy in the left calcarine gyrus and cerebellum, executive, and visuospatial dysfunction were related to the atrophy in thalamus (27). Similar studies have proven that multi- 
atrophic areas, and cognitive dysfunction also existed in MSA-P patients. Evidence from multiple linear regression analyses proved that executive dysfunction, memory disorder, and the general mental state correlated with the atrophy in the frontal and striatum, temporal lobe, and cerebellum respectively. Due to high independence of those correlations, the cognitive dysfunction of the MSA patients might be induced by the lesions in the cerebral cortex, cerebellum or subcortical circuit individually (25).

Of note, in other studies, neuropsychological and [(18)F]deoxyglucose (FDG) PET scans were performed to check the metabolic status of the MSA patients. The findings showed that patients in the early stage had a metabolism deficit in the frontal cortex, anterior cerebellar hemisphere, and vermis instead of the basal ganglia. Along with the development of these diseases, the hypometabolism was further spread to the frontal and parieto-temporal cortices, the bilateral caudate nucleus, and the left posterolateral putamen; these, as well as the whole striatum. Meanwhile, the cognitive dysfunction was also aggravated from frontal lobe dominant executive disorder to various cognitive disorders. These findings indicated that the cognitive dysfunction of MSA patients in the early stage was mainly correlated with the frontal lobe and cerebellum. During the late stage, the injury of caudate nucleus and putamen might aggravate the illness via damaging the subcortical striatum-cerebral cortex pathway $(24,45)$. On the contrary, a research by Perani and his colleagues illustrated that the selective metabolic reduction was mainly in the striatum and cerebellum of MSA-P and MSA-C patients at the early stage, while the patients at the late stage usually had the hypometabolism in the frontal lobe, both of which showed no significant correlation (35).

In summary, although a series of studies have been carried out, the exact brain areas responsible for cognitive dysfunction of the MSA patients, and the underlying molecular mechanisms are still largely unknown. Current studies demonstrated that the cognitive disorders of MSA patients were derived either from the cerebral cortex, especially prefrontal lobe lesions, various lesions in the subcortical circuit loop or lesions from different regions. Moreover, cognitive dysfunction should be included in the criteria for the diagnosis of MSA.

\section{Perspectives}

Thanks to the extensive application of neuroimaging, neuropathology, and electro-neurophysiology in the MSA study, our knowledge regarding MSA has been extended greatly. However, there are several essential questions that still remain unanswered. For instance, the detailed relationship between brain morphology alteration and cognitive dysfunction needs to be clarified. In order to explore the mechanisms at the molecular level, and to identify novel special biomarkers for MSA, it is critical for researchers to establish and optimize animal models for MSA. This will lay a foundation for an early diagnosis and treatment of MSA, and provide solid evidence for involving cognitive dysfunction as standard diagnosis for MSA in the future.

\section{Acknowledgements}

Funding: Grant support from Science Research Foundation Project of the Second Hospital of Hebei Medical University, China (No. 2h2017079) and the Crosswise Project of the Second Hospital of Hebei Medical University, China (No. 303-09-02-32) is gratefully acknowledged.

\section{Footnote}

Conflicts of Interest: The authors have no conflicts of interest to declare.

\section{References}

1. Gilman S, Wenning GK, Low PA, Brooks DJ, Mathias CJ, Trojanowski JQ, Wood NW, Colosimo C, Dürr A, Fowler CJ, Kaufmann H, Klockgether T, Lees A, Poewe W, Quinn N, Revesz T, Robertson D, Sandroni P, Seppi K, Vidailhet $M$. Second consensus statement on the diagnosis of multiple system atrophy. Neurology 2008;71:670-6.

2. Graham JG, Oppenheimer DR. Orthostatic hypotension and nicotine sensitivity in a case of multiple system atrophy. J Neurol Neurosurg Psychiatry 1969;32:28-34.

3. Jellinger KA. Neuropathology of multiple system atrophy: new thoughts about pathogenesis. Mov Disord 2014;29:1720-41.

4. Fanciulli A, Wenning GK. Multiple-system atrophy. N Engl J Med 2015;372:249-63.

5. Poewe W, Mahlknecht P, Krismer F. Therapeutic advances in multiple system atrophy and progressive supranuclear palsy. Mov Disord 2015;30:1528-38.

6. Jecmenica-Lukic M, Poewe W, Tolosa E, Wenning GK. Premotor signs and symptoms of multiple system atrophy. Lancet Neurol 2012;11:361-8. 
7. Brown RG, Lacomblez L, Landwehrmeyer BG, Bak T, Uttner I, Dubois B, Agid Y, Ludolph A, Bensimon G, Payan C, Leigh NP. Cognitive impairment in patients with multiple system atrophy and progressive supranuclear palsy. Brain 2010;133:2382-93.

8. Siri C, Duerr S, Canesi M, Delazer M, Esselink R, Bloem BR, Gurevich T, Balas M, Giladi N, Santacruz P, Marti F, Tolosa E, Rubino A, Meco G, Poewe W, Pezzoli G, Wenning G, Antonini A. A cross-sectional multicenter study of cognitive and behavioural features in multiple system atrophy patients of the parkinsonian and cerebellar type. J Neural Transm (Vienna) 2013;120:613-8.

9. Stankovic I, Krismer F, Jesic A, Antonini A, Benke T, Brown RG, Burn DJ, Holton JL, Kaufmann H, Kostic VS, Ling H, Meissner WG, Poewe W, Semnic M, Seppi K, Takeda A, Weintraub D, Wenning GK. Cognitive impairment in multiple system atrophy: a position statement by the Neuropsychology Task Force of the MDS Multiple System Atrophy (MODIMSA) study group. Mov Disord 2014;29:857-67.

10. Kitayama M, Wada-Isoe K, Irizawa Y, Nakashima K. Assessment of dementia in patients with multiple system atrophy. Eur J Neurol 2009;16:589-94.

11. Köllensperger M, Geser F, Ndayisaba JP, Boesch S, Seppi K, Ostergaard K, Dupont E, Cardozo A, Tolosa E, Abele M, Klockgether T, Yekhlef F, Tison F, Daniels C, Deuschl G, Coelho M, Sampaio C, Bozi M, Quinn N, Schrag A, Mathias CJ, Fowler C, Nilsson CF, Widner H, Schimke N, Oertel W, Del SF, Albanese A, Pellecchia MT, Barone P, Djaldetti R, Colosimo C, Meco G, GonzalezMandly A, Berciano J, Gurevich T, Giladi N, Galitzky M, Rascol O, Kamm C, Gasser T, Siebert U, Poewe W, Wenning GK. Presentation, diagnosis, and management of multiple system atrophy in Europe: final analysis of the European multiple system atrophy registry. Mov Disord 2010;25:2604-12.

12. O'Sullivan SS, Massey LA, Williams DR, SilveiraMoriyama L, Kempster PA, Holton JL, Revesz T, Lees AJ. Clinical outcomes of progressive supranuclear palsy and multiple system atrophy. Brain 2008;131:1362-72.

13. Spaccavento S, Del PM, Loverre A, Craca A, Nardulli R. Multiple system atrophy with early cognitive deficits: a case report. Neurocase 2013;19:613-22.

14. Stanzani-Maserati M, Gallassi R, Calandra-Buonaura G, Alessandria M, Oppi F, Poda R, Sambati L, Provini F, Cortelli P. Cognitive and sleep features of multiple system atrophy: review and prospective study. Eur Neurol 2014;72:349-59.
15. Kawai Y, Suenaga M, Takeda A, Ito M, Watanabe H, Tanaka F, Kato K, Fukatsu H, Naganawa S, Kato T, Ito $\mathrm{K}$, Sobue G. Cognitive impairments in multiple system atrophy: MSA-C vs. MSA-P. Neurology 2008;70:1390-6.

16. Jetter W, Poser U, Freeman RB, Markowitsch HJ. A verbal long term memory deficit in frontal lobe damaged patients. Cortex 1986;22:229-42.

17. Marconi R, Antonini A, Barone P, Colosimo C, Avarello TP, Bottacchi E, Cannas A, Ceravolo MG, Ceravolo R, Cicarelli G, Gaglio RM, Giglia L, Iemolo F, Manfredi M, Meco G, Nicoletti A, Pederzoli M, Petrone A, Pisani A, Pontieri FE, Quatrale R, Ramat S, Scala R, Volpe G, Zappulla S, Bentivoglio AR, Stocchi F, Trianni G, Del DP, De Gaspari D, Grasso L, Morgante F, Santangelo G, Fabbrini G, Morgante L. Frontal assessment battery scores and non-motor symptoms in parkinsonian disorders. Neurol Sci 2012;33:585-93.

18. Hoops S, Nazem S, Siderowf AD, Duda JE, Xie SX, Stern MB, Weintraub D. Validity of the MoCA and MMSE in the detection of MCI and dementia in Parkinson disease. Neurology 2009;73:1738-45.

19. Fiorenzato E, Weis L, Falup-Pecurariu C, Diaconu S, Siri C, Reali E, Pezzoli G, Bisiacchi P, Antonini A, Biundo R. Montreal Cognitive Assessment (MoCA) and Mini-Mental State Examination (MMSE) performance in progressive supranuclear palsy and multiple system atrophy. J Neural Transm (Vienna) 2016;123:1435-42.

20. Ihara M, Okamoto Y, Takahashi R. Suitability of the Montreal cognitive assessment versus the mini-mental state examination in detecting vascular cognitive impairment. J Stroke Cerebrovasc Dis 2013;22:737-41.

21. Wáng YX. Systemic review and meta-analysis of diagnostic imaging technologies. Quant Imaging Med Surg 2016;6:615-8.

22. Ugga L, Coppola C, Cocozza S, Saracino D, Caranci F, Tuccillo F, Signoriello E, Casertano S, Di IG, Tedeschi E. Diagnostic contribution of magnetic resonance imaging in an atypical presentation of motor neuron disease. Quant Imaging Med Surg 2017;7:727-31.

23. Chang CC, Chang YY, Chang WN, Lee YC, Wang YL, Lui CC, Huang CW, Liu WL. Cognitive deficits in multiple system atrophy correlate with frontal atrophy and disease duration. Eur J Neurol 2009;16:1144-50.

24. Lyoo CH, Jeong Y, Ryu YH, Lee SY, Song TJ, Lee JH, Rinne JO, Lee MS. Effects of disease duration on the clinical features and brain glucose metabolism in patients with mixed type multiple system atrophy. Brain 2008;131:438-46. 
25. Kim JS, Yang JJ, Lee DK, Lee JM, Youn J, Cho JW. Cognitive Impairment and Its Structural Correlates in the Parkinsonian Subtype of Multiple System Atrophy. Neurodegener Dis 2015;15:294-300.

26. Messina D, Cerasa A, Condino F, Arabia G, Novellino F, Nicoletti G, Salsone M, Morelli M, Lanza PL, Quattrone A. Patterns of brain atrophy in Parkinson's disease, progressive supranuclear palsy and multiple system atrophy. Parkinsonism Relat Disord 2011;17:172-6.

27. Lee MJ, Shin JH, Seoung JK, Lee JH, Yoon U, Oh JH, Jung DS, Kim EJ. Cognitive impairments associated with morphological changes in cortical and subcortical structures in multiple system atrophy of the cerebellar type. Eur J Neurol 2016;23:92-100.

28. Ahmed Z, Asi YT, Sailer A, Lees AJ, Houlden H, Revesz T, Holton JL. The neuropathology, pathophysiology and genetics of multiple system atrophy. Neuropathol Appl Neurobiol 2012;38:4-24.

29. Trojanowski JQ, Revesz T. Proposed neuropathological criteria for the post mortem diagnosis of multiple system atrophy. Neuropathol Appl Neurobiol 2007;33:615-20.

30. Jellinger KA, Wenning GK. Multiple system atrophy:pathogenic mechanisms and biomarkers. J Neural Transm (Vienna) 2016;123:555-72.

31. Ozawa T, Paviour D, Quinn NP, Josephs KA, Sangha H, Kilford L, Healy DG, Wood NW, Lees AJ, Holton JL, Revesz T. The spectrum of pathological involvement of the striatonigral and olivopontocerebellar systems in multiple system atrophy: clinicopathological correlations. Brain 2004;127:2657-71.

32. Inoue M, Yagishita S, Ryo M, Hasegawa K, Amano N, Matsushita M. The distribution and dynamic density of oligodendroglial cytoplasmic inclusions (GCIs) in multiple system atrophy: a correlation between the density of GCIs and the degree of involvement of striatonigral and olivopontocerebellar systems. Acta Neuropathol 1997;93:585-91.

33. Papp MI, Lantos PL. The distribution of oligodendroglial inclusions in multiple system atrophy and its relevance to clinical symptomatology. Brain 1994;117:235-43.

34. Wenning GK, Tison F, Ben SY, Daniel SE, Quinn NP. Multiple system atrophy:a review of 203 pathologically proven cases. Mov Disord 1997;12:133-47.

35. Perani D, Bressi S, Testa D, Grassi F, Cortelli P, Gentrini S, Savoiardo M, Caraceni T, Fazio F. Clinical/metabolic correlations in multiple system atrophy. A fludeoxyglucose F 18 positron emission tomographic study. Arch Neurol 1995;52:179-85.
36. Wang Y, Lü C, Ye Z. Alpha-synuclein immunoreactivity and ultrastructural study of glial cytoplasmic inclusions in multiple system atrophy. Chin Med J (Engl) 2002;115:1491-5.

37. Middleton FA, Strick PL. Basal ganglia and cerebellar loops:motor and cognitive circuits. Brain Res Brain Res Rev 2000;31:236-50.

38. Middleton FA, Strick PL. Basal ganglia output and cognition:evidence from anatomical, behavioral, and clinical studies. Brain Cogn 2000;42:183-200.

39. Parent A, Hazrati LN. Functional anatomy of the basal ganglia. II. The place of subthalamic nucleus and external pallidum in basal ganglia circuitry. Brain Res Brain Res Rev 1995;20:128-54.

40. Fiez JA, Petersen SE, Cheney MK, Raichle ME. Impaired non-motor learning and error detection associated with cerebellar damage. A single case study. Brain 1992;115 Pt 1:155-78.

41. Temel Y, Blokland A, Steinbusch HW, VisserVandewalle V. The functional role of the subthalamic nucleus in cognitive and limbic circuits. Prog Neurobiol 2005;76:393-413.

42. Martín Noguerol T, Barousse R, Socolovsky M, Luna A. Quantitative magnetic resonance (MR) neurography for evaluation of peripheral nerves and plexus injuries. Quant Imaging Med Surg 2017;7:398-421.

43. Winston GP. The potential role of novel diffusion imaging techniques in the understanding and treatment of epilepsy. Quant Imaging Med Surg 2015;5:279.

44. Hagmann P, Thiran JP, Jonasson L, Vandergheynst P, Clarke S, Maeder P, Meuli R. DTI mapping of human brain connectivity: statistical fibre tracking and virtual dissection. Neuroimage 2003;19:545-54.

45. Lehéricy S, Ducros M, Van de Moortele PF, Francois C, Thivard L, Poupon C, Swindale N, Ugurbil K, Kim DS. Diffusion tensor fiber tracking shows distinct corticostriatal circuits in humans. Ann Neurol 2004;5 5:522-9.

46. Mori S, Kaufmann WE, Davatzikos C, Stieltjes B, Amodei L, Fredericksen K, Pearlson GD, Melhem ER, Solaiyappan M, Raymond GV, Moser HW, van Zijl PC. Imaging cortical association tracts in the human brain using diffusion-tensor-based axonal tracking. Magn Reson Med 2002;47:215-23.

47. Prats-Galino A, Soria G, de Notaris M, Puig J, Pedraza S. Functional anatomy of subcortical circuits issuing from or integrating at the human brainstem. Clin Neurophysiol 2012;123:4-12.

48. Stieltjes B, Kaufmann WE, van Zijl PC, Fredericksen K, 
Pearlson GD, Solaiyappan M, Mori S. Diffusion tensor imaging and axonal tracking in the human brainstem. Neuroimage 2001;14:723-35.

49. Brown RG, Marsden CD. 'Subcortical dementia': the neuropsychological evidence. Neuroscience 1988;25:363-87.

50. Parent A, Hazrati LN. Functional anatomy of the basal ganglia. I. The cortico-basal ganglia-thalamo-cortical loop. Brain Res Brain Res Rev 1995;20:91-127.

Cite this article as: Zhang L, Zhang L, Xue F, Yue K, Peng $\mathrm{H}$, Wu Y, Sha O, Yang L, Ding Y. Brain morphological alteration and cognitive dysfunction in multiple system atrophy. Quant Imaging Med Surg 2018;8(10):1030-1038. doi: 10.21037/ qims.2018.11.02
51. Schmahmann JD, Sherman JC. The cerebellar cognitive affective syndrome. Brain 1998;121:561-79.

52. Gottwald B, Wilde B, Mihajlovic Z, Mehdorn HM. Evidence for distinct cognitive deficits after focal cerebellar lesions. J Neurol Neurosurg Psychiatry 2004;75:1524-31.

53. Bürk K, Daum I, Rüb U. Cognitive function in multiple system atrophy of the cerebellar type. Mov Disord 2006;21:772-6. 